\title{
Der Abelsche Satz.
}

(Von Herrn E. Heine in Halle.)

In dem Aufsatze über die Laméschen Functionen verschiedener Ordnungen, der sich im $60^{\text {sten }}$ Bande dieses Journals findet, zeigt eine Integration durch Theile unter I, \$. 4, Gleichung $(\varepsilon)$ auf S. 266, dass das Integral

der Gleichung

$$
J=\int_{\alpha}^{\beta} \frac{d z}{(x-z) \sqrt{\psi(z)}}
$$

$$
\text { (1.) } \sqrt{\psi(x)} \frac{d(J \cdot \sqrt{\psi(x)})}{d x}=\frac{\sqrt{\psi(\alpha)}}{x-\alpha}-\frac{\sqrt{\psi(\beta)}}{x-\beta}+G(x)
$$

genügt. Hier bedeutet $\psi(x)$ eine willkürliche gànze Function, von der $G(x)$ durch die Gleichung

$$
G(x)=\int_{a}^{\beta}\left[\frac{1}{2} \frac{\psi^{\prime}(x)-\psi^{\prime}(z)}{x-z}-\frac{d}{d z} \cdot\left(\frac{\psi(x)-\psi(z)}{x-z}\right)\right] \frac{d z}{\sqrt{\psi(z)}}
$$

abhängt, so dass auch $\boldsymbol{G}(\boldsymbol{x})$ eine ganze Function von $x$ wird, deren Grad aber um zwei Einheiten geringer ist als der Grad von $\psi(x)$. Ferner bezeichnen $\alpha$ und. $\beta$ Constanten, und der Integrationsweg $z$ in $J$ kann zwischen den Grenzen $\alpha$ und $\beta$ willkürlich genommen werden, darf aber nicht durch den Punkt $x$ selbst führen. Die Quadratwurzel $\sqrt{\psi(z)}$ muss sich in den Integrationsgrenzen $\alpha$ bis $\beta$ continuirlich ändern, und $\sqrt{\psi(\alpha)}$ und $\sqrt{\psi(\beta)}$ auf der rechten Seite von (1.) stellen den Anfangs- und Endwerth dieser Quadratwurzel vor.

In der erwähnten Abhandlung wurde S. 267 bemerkt, dass vermittelst dieser Formel sich der Abelsche Satz beweisen lässt, zunächst der speciellere, wie ihn Jacobi ${ }^{*}$ ) ausgesprochen hat; der Beweis soll an dieser Stelle mitgetheilt werden.

Es sei $\psi(z)$ eine gegebene ganze Function vom Grade $2 n+1$, die durch $z$ getheilt für $z=0$ gleich 1 werden mag; die Wurzeln der Gleichung $\psi(z)=0$ heissen $r_{1}, r_{1}$, elc. $r_{2 n}$, und werden als sämmtlich verschieden vorausgesetzt. Ferner sei $w(z)$ eine ganze, durch $z$ theilbare Function $n+1^{\text {ten }}$ Grades, in welcher der Coefficient von $z^{n+1}$ die Einheit ist, und $a$ eine Constante. Um diese Bestimmungen besser vor Augen zu haben, kann man

*) Crelle, Journal f. Mathematik Bd. XXX, S. 121: Ueber die Additionstheoreme der Abelschen Integrale zweiter und dritter Gattung. 
sich der Gleichungen bedienen :

$$
\begin{aligned}
& \psi(z)=z\left(b_{1} z^{2 n}+b_{2} z^{2 n-1}+\text { etc. }+b_{2 n} z+1\right)=\left(z-r_{0}\right)\left(z-r_{1}\right) \ldots\left(z-r_{2 n}\right), \\
& w(z)=z\left(z^{n}+c_{1} z^{n-1}+\text { etc. }+c_{n-1} z+c_{n}\right) .
\end{aligned}
$$

Ausserdem setze man

(2.) $(w(z))^{2}-a^{2} \psi(z)=z f(z)=z\left(z-\alpha_{1}\right)\left(z-\alpha_{2}\right) \ldots\left(z-\alpha_{2 n+1}\right)$.

Es mag nun die Function $w$, durch continuirliche Veränderung ihrer Constanten $c$, sich in eine Function $\boldsymbol{W}(\boldsymbol{z})$ verwandeln, welche dieselben Eigenschaften besitzt, die oben $w$ beigelegt wurden; zu gleicher Zeit soll $a$ in $A$ übergehen. Endlich setze man noch

(3.) $\quad(\boldsymbol{W}(\boldsymbol{z}))^{2}-\boldsymbol{A}^{2} \psi(z)=\boldsymbol{z} \boldsymbol{F}(\boldsymbol{z})=\boldsymbol{z}\left(\boldsymbol{z}-\boldsymbol{\beta}_{1}\right)\left(z-\boldsymbol{\beta}_{2}\right) \ldots\left(z-\boldsymbol{\beta}_{2 n+1}\right)$, und denke sich unter $\beta_{1}, \beta_{2}$, etc. diejenigen Wurzeln von $F(z)=0$, in welche respective $\alpha_{1}, \alpha_{2}$, etc. continuirlich übergegangen sind.

Man bilde nun $2 n+1$ Integrale $J$, die $J_{1}, J_{2}$, etc. heissen, indem man successive $\alpha$ und $\beta$ zugleich Indices respective 1,2 , etc. anhängt. Der Integrationsweg $z$ in $J_{1}$ ist zunächst der Weg, auf welchem $\alpha_{1}$ in $\beta_{1}$ übergeht, und diesen hält man als Integrationsweg z beim Beweise fest, kann ihn aber, wenn erst das Resultat festgestellt ist, auf verschiedene Art abändern. Man muss (s. o.) annehmen, dass $x$ nicht im Wege $z$ liegt, weil sonst das Integral $J_{1}$ seine Bedeutung verliert. Bei Festsetzung des Zeichens von $\sqrt{\psi(z)}$ nehmen wir an, um Weitläufigkeiten im Ausdrucke zu vermeiden, dass von den Wegen, auf denen $a$ in $A$ und $\alpha_{1}$ in $\beta_{1}$ übergeht, der erstere nicht durch Null, der zweite durch kein $r$ führt. Indem man in üblicher Weise die Wege, wenn sie diese Punkte treffen sollten, unendlich wenig abändert, kann man das Verlangle erreichen. Der Quadratwurzel $\sqrt{\psi(z)}$ giebt man nun als Anfangswerth denjenigen, welcher der Gleichung $w\left(\alpha_{1}\right)=a \sqrt{\psi\left(\alpha_{1}\right)}$ entspricht; im weiteren Verlaufe wird dann $\sqrt{\psi(z)}$ überall bestimmt sein, trifft also, da ausserdem $w, \alpha, a$ continuirlich in $W, \beta, A$ übergehen, mit einem solchen Werthe in $\beta_{1}$ ein, der $W\left(\beta_{1}\right)=A \sqrt{\psi\left(\beta_{1}\right)}$ entspricht. Aehnliches gilt auch für $J_{2}, J_{3}$, etc.

Bildet man für jedes $J$ eine Gleichung wie (1.), addirt diese Gleichungen und setzt die Summe der $2 n+1$ Integrale $J$ gleich $S$, so erhält man

$$
S=J_{1}+J_{2}+\text { etc. }+J_{2 n+1},
$$

(4.) $\quad \sqrt{\psi(x)} \frac{d(S \cdot \sqrt{\psi(x)})}{d x}=\sum_{\alpha} \frac{\sqrt{\psi(\alpha)}}{x \rightarrow \alpha}-\sum_{\beta} \frac{\sqrt{\psi(\beta)}}{x-\beta}+H(x)$,

wo $H$ eine Summe von Functionen wie $G$, also wiederum eine ganze Function von $x$ höchstens vom $2 n-1^{\text {ten }}$ Grade vorstellt, und die Summenzeichen selbstverständlich eine Summation über alle $2 n+1$ Werthe respective $\alpha$ oder $\beta$ andeuten. 
Bezeichnet $\Theta(x)$ irgend eine ganze Function höchstens $2 n^{\text {ten }}$ Grades, so ist nach der Lagrangeschen Formel

$$
\sum_{\alpha} \frac{\Theta(\boldsymbol{\alpha})}{(\boldsymbol{x}-\boldsymbol{\alpha}) f^{\prime}(\boldsymbol{\alpha})}=\frac{\Theta(\boldsymbol{x})}{f(\boldsymbol{x})}
$$

war $\Theta(x)$ von höherem Grade, so giebt die linke Seite nur den Rest, welcher bei Division von $\Theta(x)$ durch $f(x)$ übrig bleibt. Wir bezeichnen ihn durch $\boldsymbol{R}[\Theta(x)]: f(x)$.

Da nun $a \sqrt{\psi(\alpha)}$ gleich $w(\alpha)$ war, so hat män

$$
\sum_{\alpha} \frac{\sqrt{\psi(\alpha)}}{x-\alpha}=\frac{1}{a} R\left[w(x) f^{\prime}(x)\right]: f(x)
$$

Die rechte Seite dieser Gleichung lässt sich durch (2.) noch weiter transformiren; fügt man die Argumente $x$ vorläufig den Functionen nicht hinzu, so ergiebt sich aus (2.)

$$
\begin{aligned}
x f^{\prime} & =2 w w^{\prime}-a^{2} \psi^{\prime}-f, \\
\boldsymbol{R}\left[w f^{\prime}\right]: f & =\boldsymbol{R}\left[\frac{2 w^{2} w^{\prime}-a^{2} w \psi^{\prime}}{x}\right]: f,
\end{aligned}
$$

und selzt man für $w^{2}$ seinen Werth $x f+a^{2} \psi$

$$
\frac{1}{a} R\left[w f^{\prime}\right]: f=a R\left[\frac{2 \psi w^{\prime}-w \psi^{\prime}}{x}\right]: f \text {. }
$$

Der Grad der ganzen Function, welche rechts durch $f$ zu theilen ist, beträgt $3 n$, derjenige des Divisors $f$ aber $2 n+1$; der Rest, wèlchen der Bruch giebt und den das Zeichen $\boldsymbol{R}$ andeutet, ist also gleich dem Bruche weniger einer ganzen Function $\gamma$ vom $n-1^{\text {ten }}$ Grade, und man hat

$$
\sum_{a} \frac{\sqrt{\psi(\alpha)}}{x-\alpha}=a \frac{2 \psi w^{\prime}-w \psi^{\prime}}{x}-\gamma
$$

so wie einen ähnlichen Ausdruck, welcher $\gamma_{1}$ als ganze Function enthalten mag, für die Summe nach $\beta$ in (4.). Zieht man noch $\boldsymbol{H}$ mit $\gamma$ und $\gamma_{1}$ in eine einzige Function $K(x)$ zusammen, so dass $K(x)$ wiederum eine ganze Function höchstens vom $2 n-1^{\text {ten }}$ Grade wird, so entsteht aus (4.)

$$
\text { (5.) } \frac{d(S \cdot \sqrt{\psi(x)})}{d x}=a \cdot \frac{2 \psi(x) w^{\prime}(x)-w(x) \psi^{\prime}(x)}{x f(x) \sqrt{\psi(x)}}-A \cdot \frac{2 \psi(x) W^{\prime}(x)-W(x) \psi^{\prime}(x)}{x F(x) \sqrt{\psi(x)}}+\frac{K(x)}{\sqrt{\psi(x)}} \text {. }
$$

Das erste und zweite Glied der rechten Seite sind ebenso wie die linke Seite vollständige Differentialquótienten nach $x$; integrirt man nach $x$ von $x=r_{0}$ an, und selzt

so findet man

$$
Z(x)=\int_{r_{*}} \frac{K(x) d x}{\sqrt{\psi(x)}}
$$




$$
\text { (6.) } \quad S \sqrt{\psi(x)}=\log \frac{W(x)+A \sqrt{\psi(x)}}{W(x)-A \sqrt{\psi(x)}}-\log \frac{w(x)+a \sqrt{\psi(x)}}{w(x)-a \sqrt{\psi(x)}}+Z(x),
$$

also den Abelschen Satz, wenn man zeigen kann, dass $Z(x)$ verschwindet.

Um dies zu beweisen, geht man davon aus, dass, wie unten gezeigt werden wird, $Z(x)$, auf welchem Wege man auch nach $x$ von $r_{0}$ an integrirt haben möge, für $x=r_{11}, r_{1}$, etc. $r_{2 n}$ gleich Null wird. Ist dies festgestellt, so gestaltet der Beweis sich auf folgende Art: Das Integral $Z$ ist eine solche Function von $x$, welche in einem Punkte $x$ nicht immer denselben Werth $v$ besitzt, sondern bei Veränderung des Integrationsweges $x$ auch den entgegengesetzten Werth $-v$ annimmt, und endlich die Werthe $v$ und $-v$, vermehrt um positive oder negative Vielfache von den Grössen $\boldsymbol{Z}\left(\boldsymbol{r}_{10}\right), \boldsymbol{Z}\left(\boldsymbol{r}_{1}\right)$, etc. $\boldsymbol{Z}\left(\boldsymbol{r}_{2 n}\right)$. Da die letzteren Ausdrücke nach der Voraussetzung sämmtlich Null sind, so ist $(Z(x))^{2}$ eine continuirliche einwerthige Function von $x$, die nur für $x=\infty$ unendlich wird, d. h. es ist eine ganze Function von $x$. Diese muss durch $\psi(x)$ theilbar sein, da $Z(x)$ für $x=r_{1}, r_{1}$, etc. $r_{2 n}$ verschwindet; also hat $\boldsymbol{Z}(\boldsymbol{x})$ die Form $Q \sqrt{\psi(x)}$, wo $\boldsymbol{Q}$ nur noch eine Quadratwurzel aus einer ganzen Function sein kann. Hieraus folgt

$$
\begin{gathered}
\frac{K(x)}{\sqrt{\psi(x)}}=\frac{K(x) Q}{Q \sqrt{\psi(x)}}=\frac{d\left(Q_{\bigvee} \sqrt{\psi(x)}\right)}{d x}, \\
2 K(x) Q d x=d\left(Q_{\sqrt{\psi(x})}\right)^{2}=d\left(Q^{2} \psi(x)\right) ;
\end{gathered}
$$

also ist $Q$ eine ganze Function von $x$. Soll diese vom Grade $m$ sein, so wird $d\left(Q \sqrt{\psi^{\prime}(x)}\right)$ jedenfalls von der Form $\frac{L d x}{\sqrt{\psi(x)}}$, wo $L$ ganz und vom Grade $2 n+m$ ist. Es kann $L$ also nicht, wie es geschehen soll, mit $K(x)$ übereinstimmen, da dieses nur den Grad $2 n-1$ erreicht, d. h. es ist $L=0, K=0$, folglich $\boldsymbol{Z}(\boldsymbol{x})=\mathbf{0}$.

Um schliesslich zu zeigen, dass $Z(x)$ wirklich für $x=r_{0}, r_{1}$, etc. $r_{2 n}$ verschwindet, wie so eben vorausgesetzt war, heben wir folgende Punkte hervor:

a) Ein bestimmtes Integral kaun bekanntlich noch eine Bedeutung behalten, wenn auch die zu integrirende Function, die $\vartheta(x)$ heissen mag, an einer Stelle $\xi$ des Integrationsweges $\mathscr{C}$ discontinuirlich wird. Stellt $a b$ den Integrationsweg $x$ vor, also $\xi$ einen Punkt dieses Weges, und sind $\xi-\varepsilon$ und $\xi+\eta$ zwei Punkte des Weges $a b$, die $\xi$ sehr nahe liegen, der eine zur Linken von $\xi$, nach $a \mathrm{zu}$, der andere zur Rechten, zwischen $\xi$ und $b$, so ist nämlich das Integral von $\boldsymbol{\vartheta}(\boldsymbol{x}) d x$, von $a$ bis $b$ über $a b$ integrirt, gleich der Grenze von der Summe der beiden Integrale von $a$ bis $\xi-\varepsilon$ und von $\xi+\eta$ 
bis $b$, die Grenze für verschwindende $\delta$ und $\eta$ genommen. Existiren solche Grenzen, so hat das Integral von $a$ bis $b$ über $a b$ noch einen Werth. Solche Grenzen werden wir abgekürzt als Integrale von $a$ bis $\xi-0$, oder von $\xi+0$ bis $b$ bezeichnen.

b) Sind für alle Werthe, die $x$ auf dem Wege ab annimmt, zwei Functionen $\vartheta(x)$ und $\varphi(x)$ continuirlich und gleich, so wird

$$
\int_{a}^{b} \boldsymbol{\vartheta}(x) d x=\int_{a}^{b} \varphi(x) d x
$$

findet an dem Endpunkte $b$ die Gleichheit $\boldsymbol{\varphi}(\boldsymbol{x})=\varphi(x)$ nicht mehr statt, was geschehen kann wenn diese Functionen dort unstätig werden, so findet doch die obige Integralgleichung statt, vorausgesetzt dass die Integrale einen Werth behalten, da sie dann Integrale von $a$ bis $b-0$ werden. Dasselbe gilt, wenn $\vartheta$ und $\varphi$ am Anfangspunkte $a$ ungleich werden, folglich auch, nach (a), wenn in einem mittleren Punkte $\xi$ die Ungleichheit eintritt: immer noch werden die obigen Integrale übereinstimmen, wenn sie überhaupt einen Werth besitzen.

Auf (5.) angewandt zeigen diese Bemerkungen, dass die Integrale der beiden Seiten dieser Gleichung, auf einem beliebigen Wege $a b$ nach $x$ genommen, mag auch der Weg $x$ durch die Curven $z$ führen, wenn die Integrale überhaupt existiren, auch gleich sind, obgleich das Bestehen von (5.) ursprünglich voraussetzte, es liege $x$ nicht in gewissen Curven, den Integrationswegen $z$.

c) Ist $\Theta(x)$ irgend ein einwerthiges Integral von $\boldsymbol{\vartheta}(\boldsymbol{x}) \boldsymbol{d x}$, welches auf dem Wege $a b$ stetig bleibt, und höchstens in dem Punkte $\xi$ dieses Weges (für andere Wege $a b$ können gleichfalls Punkte wie $\xi$ existiren) keinen bestimmten Werth besitzt, so wird das Integral von $\vartheta(x) d x$, über diesen Werth $a b$ genommen, gleich $\Theta(b)-\Theta(a)-(\Theta(\xi+0)-\Theta(\xi-0))$.

Stellt $\boldsymbol{\vartheta}(\boldsymbol{x})$ die linke Seite von (5.) vor, so besitzen wir in $S . \sqrt{\psi(x)}$ ein solches Integral $\Theta(x)$, wenn zunächst solche Wege $x$ ausgeschlossen werden, die einzeln oder von denen mehrere zusammengenommen einen oder mehrere Punkte $r$ umkreisen; das Zeichen von $\sqrt{\psi(x)}$ ist dann ein vollständig bestimmtes, sobald es, wie wir immer annehmen, für $x=r_{0}, \mathrm{~d}$. h. für ein $x$, welches $r_{0}$ unendlich nahe liegt, gegeben war. Zerlegt man nämlich $\boldsymbol{S}$ wieder in seine Bestandtheile $J$, so sieht man, dass $S \cdot \sqrt{\psi(x)}$ sich mit $x$ continuirlich ändert, so lange $x$ nicht einen Weg $z$ durchschneidet, wie nahe auch $x$ dem Wege z liegt. Beim Durchschneiden eines Weges $z$ ändert sich aber $S \cdot \sqrt{4 !(x)}$ plötzlich um $\pm 2 \pi i$, welcher Werth in obiger Formel dem Ausdrucke $\Theta(\xi+0)-\Theta(\xi-0)$ entsprechen würde. In der That ergiebt die für solche 
Untersuchungen übliche Behandlung des Integrales

$$
\int \frac{d z}{(x-z) \sqrt{\psi(z)}}
$$

dass es, über einen kleinen Kreis mit dem Mittelpunkte $x$ integrirt, $\pm \frac{2 \pi i}{\sqrt{\psi(x)}}$ wird. Diesen Weg z, den kleinen Kreis, kann man aber, ohne den Werth des Integrales zu ändern, so auseinander ziehen, dass er ein schmaler länglicher Streifen wird, der den Punkt $x$ noch immer umschliesst, und dass man, die Begrenzung des Streifens durchlaufend, den Punkt $x$ einmal unendlich nahe zur Rechten, das andere Mal zur Linken hat. Das Integral zwischen zwei beliebigen Punkten der Contour dieses Streifens genommen, das eine Mal, indem der Integrationsweg zur Rechten des Punktes $x$ ihm unendlich nahe vorbeigeht, das andere Mal, indem der Weg, dem ersten unendlich nahe, zur Linken des Punktes $x$ ihm unendlich nahe vorbeigeht, giebt also Werthe, die sich um $\pm \frac{2 \pi i}{\sqrt{\psi(x)}}$ unterscheiden.

Es wird also der Werth des Integrales der linken Seite von (5.) auf dem Wege $a b$, dessen Anfangspunkt $a$ der Punkt $r_{i v}$, dessen Endpunkt $b$ der Punkt $x$ ist, wenn ein Punkt $\xi$ anf $a b$ nicht existirt, wenn also $a b$ einen Integrationsweg $z$ nicht schneidet, $S \sqrt{\psi(x)}$, wenn $a b$ ihn einmal schneidet, $S \sqrt{\psi(x)} \pm 2 \pi i$. Allgemein, wenn ab die Integrationswege $z$ mehrfach schneidet, so wird das Integral der linken Seite von (5.) gleich $S \sqrt{\psi(x)}$ vermehrt um ganze Vielfache von $\pm 2 \pi i$, deren Anzahl sich auch sofort nach dem Obigen angeben lässt, sobald man den Weg $x$ kennt. Darf der Weg $x$ die Punkte $r$ umkreisen, so wird, je nach der Anzahl der Umkreisungen, $\sqrt{\psi(x)}$ das eine oder andere Zeichen zu geben sein.

D) Der Ausdruck auf der Rechten in (5.) zerfällt in zwei Theile. Der eine, $K$ enthaltende, giebt das endliche Integral $Z(x)$, dieses auf dem Wege $a b$ von $a=r_{0}$ bis $b=x$ genommen.

e) Setzt man

$$
P=\frac{W(x)+A \sqrt{\psi(x)}}{w(x)-a \sqrt{\psi(x)}} \cdot \frac{w(x)-a \sqrt{\psi(x)}}{W(x)+A \sqrt{\psi(x)}},
$$

so ist der andere Theil der rechten Seite von (5.) gleich

$$
\frac{1}{P} \frac{d P}{d x} \text {. }
$$

Heisst das Integral nach $x$, auf einem willkürlichen Wege von $x=r_{0}$ an genommen, $\log P$, so ist es, auf einem anderen Wege genommen, $\log P$ vermehrt um ganze Vielfache von $\pm 2 \pi i$. 
f) Da für $x=r_{0}, r_{1}$, etc. $r_{2 n}$ sowohl $S \sqrt{\psi(x)}$ verschwindet, als auch $\log P$ gleich einem ganzen Vielfachen von $\pm 2 \pi i$ oder Null wird, so ist $Z\left(r_{0}\right)$, $\boldsymbol{Z}\left(\boldsymbol{r}_{\mathbf{1}}\right)$, etc. $\boldsymbol{Z}\left(\boldsymbol{r}_{2 n}\right)$ entweder Null, wie behauptet war, oder ein ganzes Vielfache von $\pm 2 \pi i$, also dann wenigstens $\pm 2 \pi i$. Dies ist aber unmöglich. Sind nämlich die Integrationswege * klein, so werden auch, wenn man

$$
K(x)=g_{0}+g_{1} x+g_{2} x^{2}+\cdots+g_{2 n-1} x^{2 n-1}
$$

setzt, die Constanten $g$ sehr klein sein müssen. Da bei Integration auf einem beliebigen Wege $x$, immer

$$
\int \frac{x^{m} d x}{\sqrt{\psi(x)}}
$$

endlich bleibt, so wird daher $Z(x)$ klein bleiben müssen, folglich nicht $2 \pi i$ sein können.

Sind aber die Integrationswege $z$ nicht hinlänglich klein, so kann man sie theilen, indem man zwischen die $\alpha$ und $\beta$ ähnliche Grenzen in beliebiger Anzahl einschiebt. Für jedes so entstehende $Z$ weist mán wie oben nach, dass es verschwindet; es verschwindet also auch die Summe der $Z$, und man. findet den Abelschen Satz

$$
S \cdot \sqrt{\psi(x)}=\log P \text {. }
$$

Die Bedeutung des $\log P$ ist aus dem Vorhergehenden klar. Da nämlich $K$ in (5.) verschwindet, so wird (nach $\mathfrak{c}$ ) durch Integration nach $x$ auf irgend einem Wege $x$, welcher keinen Weg $z$ durchschneidet, genau $S \sqrt{\psi(x)}$ erhalten, also immer derselbe Werth, und noch dazu mit demselben Zeichen, wenn man jedesmal den Weg $x$ gleich oft Punkte $r$ umkreisen lässt. Andererseits ist $\log P$ jedes $M a l=\int \frac{d P}{P}$, das Integral über denselben Weg $x$ genommen, den man bei Integration der linken Seite wählte. Es ist also

$$
\log P=\int \frac{d P}{P}
$$

der Werth, welchen man findet, wenn man auf irgend einem Wege $x$ integrirt, der keinen Weg schneidet, und auf dem $\sqrt{\psi(x)}$ dasselbe Endzeichen erreicht, welches diese Grösse auf der linken Seite, in $S \sqrt{\psi(x)}$ hat; welchen von diesen unendlich vielen Wegen man auch wählt, immer findet man denselben Werth für den Logarithmus.

Halle, im Junì 1862. 